\title{
Chirality in metric spaces
}

\section{In memoriam Michel Deza}

\author{
Michel Petitjean ${ }^{1,2}$
}

Received: 9 February 2017 / Accepted: 24 August 2017 / Published online: 8 September 2017

(C) The Author(s) 2017. This article is an open access publication

\begin{abstract}
A definition of chirality based on group theory is presented. It is shown to be equivalent to the usual one in the case of Euclidean spaces, and it permits to define chirality in metric spaces which are not Euclidean.
\end{abstract}

Keywords Chirality $\cdot$ Symmetry $\cdot$ Metric space $\cdot$ Distance

\section{Foreword}

Michel Deza became a recognized worldwide expert in distances since 2006 when he published with his wife Elena his remarkable Dictionary of distances [3], followed by four editions of his comprehensive Encyclopedia of distances, the most recent one containing 756 pages [6]. Michel did not understand why so few international conferences on distances were organized, although the fundamental importance and the practical role of distances are obvious in many fields of sciences. It is why he requested me to organize one with him. Thus, with the help of Krassimir Markov we organized the MDA 2012 conference in Varna, which attracted the mathematicians and scientists from 23 countries [5]. This success must undoubtly be attributed to the high reputation of Michel, not to me.

Less known is the involvement of Michel in the ISA (International Symmetry Association). He co-edited with Egon Schulte in 2011 a full volume of nearly 500 pages in the journal Symmetry: Culture and Science [4], of which the boards coincide with those of the ISA. Then, in 2013 Michel entered in the ISA Advisory Board.

\footnotetext{
$凶$ Michel Petitjean

michel.petitjean@univ-paris-diderot.fr; petitjean.chiral@gmail.com

1 MTi, INSERM UMR-S 973, University Paris 7, Paris, France

2 E-pôle de génoinformatique, Institut Jacques Monod, CNRS UMR 7592 Paris, France
} 
While the role of groups in symmetry is recognized since a long, the role of distances is often neglected. Generally, the encountered definitions of symmetry set a priori the existence of some group structure, although it was proved that defining symmetry needs first the existence of a metric space, groups appearing as a consequence of several basic requirements to get a safe unifying definition of symmetry [13]. In fact, this unifying definition, which works in the Euclidean case (including color constraints) and for relations, functions, distributions, matrices, graphs, strings, and for various patterns, led to five groups (including two group actions), but none of them is a prerequisite.

A major historical consequence of this neglected role of distances is that chirality, i.e. the lack of indirect symmetry, was related since a long time to the existence of some orientable space (at least locally). This orientability condition can be relaxed: chirality can be defined in any metric space, orientable or not, provided that it is based on the unifying symmetry definition mentioned above. The proof is presented in the present paper. Its deep content was published in 2010 [14], and is reproduced here with the kind permission of Symmetrion. A variant is possible via the use of conjugacy classes, but it would lead to similar conclusions.

But there is more. Since the end of the nineteenth century, many scientists attempted to measure quantitatively chirality rather than defining it as a logical parameter (i.e. an object is chiral or is not chiral) [11]. These attempts failed except one of them [10,12] (see also section 2.9 in [11]), because this latter is based on an appropriate choice of distance. Again, Michel was right by pointing out the crucial role of distances. Furthermore, this quantification of chirality is the source of several optimization problems: characterize the most chiral object within various classes of objects. Some were recently solved [15], but some remain open [1].

\section{Introduction}

The terms chiral and chirality were introduced by Lord Kelvin [17]: I call any geometrical figure, or group of points, chiral, and say that it has chirality if its image in a plane mirror, ideally realized, cannot be brought to coincide with itself.

Following the definition above, chirality is understood as a lack of mirror symmetry, and achirality means the existence of mirror symmetry. This kind of symmetry was known several millenaries ago: a number of ancient art images exhibiting mirror symmetry can be found in the book of Darvas [2]. Weyl [19] related mirror symmetry to improper rotations, and it is now widely accepted that an object in the Euclidean space is achiral if it is identical to one of its images through an indirect isometry, an indirect isometry being the composition of translations, rotations, and of an odd number of mirror reflections. One of the simplest examples of chiral figures in the plane is any non-isosceles and non-degenerate triangle: it cannot be superimposed by composition of translations and planar rotations to its image generated by reflection around an arbitrary line (here, the mirror is a line). Conversely, any isosceles triangle is achiral in the plane. However, chiral triangles in the plane are achiral in the 3dimensional space because it always exists a translation and a spatial rotation perfectly superposing the triangle on its image through an arbitrary plane (here, the mirror is a plane). In chemistry, chirality of molecules has a special importance due to its relation 
with optical phenomena, and involves sometimes complicated situations with flexible molecules. Nevertheless, it is still based on geometrical chirality in the 3-dimensional Euclidean space.

Clearly, the modern definition of chirality needs that we consider objects in the $d$-dimensional Euclidean space. A practical consequence of the definition is that a subdimensional object is achiral because it is identical to its image reflected in the $(d-1)$-hyperplane containing the object: e.g., a planar object in the 3-dimensional space is achiral, and a chiral 3-dimensional object plounged in the 4-dimensional space becomes achiral. Thus, the actual definition of chirality is intimately related to the dimension of the Euclidean space. In this paper, we propose a general definition of chirality which does not need any Euclidean space structure, and we show that it is equivalent to the usual definition in the case of a Euclidean space. We assume further in the text that a "non-Euclidean space" is just a space which is not Euclidean, and it is not assumed to have necessarily a geometric structure such as the one of hyperbolic spaces or other non-Euclidean spaces encountered in differential geometry and physics. In fact, the minimal geometric structure that we assume is induced by the existence of a distance, i.e. we work in metric spaces. The Euclidean space is also a metric space. Achirality is a particular instance of symmetry, so that we need a general definition of symmetry. The unifying one of Petitjean [13] is retained: an object is a function having its input argument in the metric space, and objects are transformed via isometric transforms over the elements of this metric space. These isometries have a group structure. Thus, the idea is to relate the definition of achirality to the properties of the group. A chiral object is just an object which is not achiral.

\section{Group structure}

Let $G$ be a group and $e$ its neutral element. Since there is no ambiguity, the symbol of the group operation will be omitted in expressions. The product of an element $a$ of $G$ by itself is noted $a^{2}$ and the inverse of $a$ is noted $a^{-1}$. Conventionnally, $a^{0}=e$. We define the set $G^{+}$as the subset of $G$ generated by products of squared elements of $G$.

Definition $1 G^{+} \stackrel{\text { def }}{=}\left\{r \in G / \exists k \in \mathbb{N}^{*}, \exists a_{i} \in G, i=1, \ldots, k, r=\prod_{i=1}^{k} a_{i}^{2}\right\}$.

Theorem $1 G^{+}$is a subgroup of $G$. It is called the direct subgroup of $G$.

Proof $G^{+}$is not empty because $e \in G$, and $G^{+}$is closed under the group operation and is closed under inversion.

Now we define $G^{-}$as being the complement of $G^{+}$to $G$.

Definition $2 G^{-} \stackrel{\text { def }}{=} G \backslash G^{+}$.

$G^{-}$may be empty. E.g., the cyclic group isomorphic to $(\mathbb{Z} / n \mathbb{Z},+)$, when $n$ is odd, is such that $G^{+}=G$ and $G^{-}=\varnothing$ because $\forall p \in\{0, \ldots, n-1\}$, either $a^{p}=a^{p / 2} a^{p / 2}$ when $p$ is even, or $a^{p}=a^{(p+n) / 2} a^{(p+n) / 2}$ when $p$ is odd. However, when $n$ is even, $G^{+}$contains the $n / 2$ even powers of the generator $a$, and $G^{-}$contains its $n / 2$ odd powers.

We assume further that $G^{-}$is not empty. 
Lemma $1 \forall r \in G^{+}$and $\forall q \in G^{-}, r q \in G^{-}$and $q r \in G^{-}$.

Proof If we assume that $r q \in G^{+}$, then, multiplying on the left by $r^{-1}$ which is in $G^{+}$ would mean that $q \in G^{+}$, which is impossible. Same proof for $q r$ by multiplication on the right by $r^{-1}$.

So, the product of two elements of $G^{+}$is in $G^{+}$, the product of an element of $G^{+}$ by an element of $G^{-}$is in $G^{-}$and conversely. In general, the product of two elements of $G^{-}$may be either in $G^{+}$or in $G^{-}$.

We also introduce the following Definition 3, which will be useful later.

Definition 3 When existing, the involutions of $G^{-}$are called mirrors, i.e. a mirror is an element of $G^{-}$which is its own inverse.

\section{Definition of chirality}

We refer to the general framework needed to define symmetry according to Petitjean [13]: $E$ being a metric space and $\delta$ being its associated distance, $F$ is the group of all bijections of $E$ onto $E$ preserving $\delta$. This group $F$ operates on $E$. Its neutral element is $I_{F}$. The elements of $F$ are isometries (in respect to $\delta$ ). Then, an object $Y$ is defined as a function having its input argument in $E$. The object $Y$ is symmetric if there is a bijection $U$ of $F$, with $U \neq I_{F}$, such that for all elements $x$ of $E, Y(U x)=Y(x)$.

Let $F^{+}$be the direct subgroup of $F$ and $F^{-}=F \backslash F^{+}$.

Definition 4 The isometries of $F^{+}$are called direct isometries, and when existing, the isometries of $F^{-}$are called indirect isometries.

Definition 5 An object having symmetry due to a direct isometry has direct symmetry, and an object having symmetry due to an indirect isometry has indirect symmetry.

Definition 6 An object having indirect symmetry is called achiral and an object having no indirect symmetry is called chiral.

By extension, when $F^{-}$is empty, a symmetric object is called chiral.

Still by extension, we propose to call chiral a non symmetric object.

\section{Euclidean spaces}

The definition of chirality given in the previous section is proved below to be equivalent with the usual one when the space is Euclidean. The distance $\delta$ is the usual Euclidean distance. The set $F$ of all isometries contains the translations, the rotations, and the orthogonal transformations composed by rotations and by an odd number of mirror inversions (i.e., the orthogonal matrices associated to these orthogonal transformations have a determinant equal to -1$)$.

The translations are all elements of $F^{+}$, because a translation of vector $t$ is always the composition of two identical translations of vector $t / 2$. Any $d$-dimensional rotation can be written as a product of $d(d-1) / 2$ Givens rotations (see Theorem 4 in "Appendix"), and each Givens rotation of angle $\theta$ is the square of two identical Givens rotations 
of angle $\theta / 2$. So, from Corollary 1 (see "Appendix") the rotations are elements of $F^{+}$. However, the orthogonal transformations composed by rotations and by an odd number of mirror inversions are elements of $F^{-}$, due to the sign of the determinant of their associated $d$-dimensional square matrix. As a particular example of these latter transformations, mirror inversions are involutions of $F^{-}$, and according to the Definition 3, can be just called mirrors.

To summarize:

- Compositions of an even number of mirrors with translations and rotations are elements of $F^{+}$.

- Compositions of an odd number of mirrors with translations and rotations are elements of $F^{-}$.

Since more than one a century the classifical definition of chirality is based on the one of Lord Kelvin [17], stating that a figure is chiral if it cannot be mapped to its mirror image by rotations and translations alone. Despite that this classical definition is rather informal, we get Theorem 2:

Theorem 2 Definition 6 is equivalent to the classical definition of chirality given for Euclidean spaces.

\section{Non Euclidean spaces}

\subsection{Strings}

The simplest situation is the case of finite strings of $n$ characters or $n$ symbols we like (bits, digits, etc.). We assume first that the $n$ positions are sequentially labelled $1, \ldots, n$. Owing to the definition of symmetry retained at the beginning of Sect. 3 , the group of isometries contains only two elements: the neutral element, represented by the identity permutation matrix $\mathbf{I}$, and the operator permuting the symbols at positions $i$ and $n+1-i$ for $i=1, \ldots, n$. The permutation matrix associated to this operator is the antidiagonal matrix $\mathbf{J}$, which is a mirror because $\mathbf{J}^{2}=\mathbf{I}$. Any relabeling of the $n$ positions would be associated to a permutation matrix $\mathbf{P}$ such that the mirror would be written $\mathbf{P J} \mathbf{P}^{\prime}$, where $\mathbf{P}^{\prime}$ is the transposed of $\mathbf{P}$, and we have again $\left(\mathbf{P J P} \mathbf{P}^{\prime}\right)\left(\mathbf{P J} \mathbf{P}^{\prime}\right)=\mathbf{I}$. It follows from our definition that palindromas are achiral, and non palindromic words are chiral. The existence of a mirror in a palindroma is obvious, although there is no Euclidean structure.

Infinite sequences of symbols in one direction have only one isometry, i.e. the neutral element. So they are never symmetric and are all chiral. Infinite sequences in both directions may be indiced by a set of signed integers isomorphic to $\mathbb{Z}$. For clarity, we assume that it is $\mathbb{Z}$ itself, and $i$ is an index taking any value in $\mathbb{Z}$. The direct subgroup $F^{+}$of isometries contains the operators $T_{k}$ translating all positions $i$ to $i+k, k$ taking any signed integer value. Its complement $F^{-}$contains the mirrors $M_{0}(j)$ permuting all positions $j+i$ with $j+1-i, j$ taking any integer value, and the mirrors $M_{1}(j)$ permuting all positions $j+i$ with $j-i, j$ taking any integer value, and the compositions of the operators $T_{k}$ with odd numbers of mirrors. So, an infinite sequence such that ...ABCABCABC... is symmetric and chiral, and an infinite 
sequence such that ...ABCCBAABCCBA... or ...ABCBAABCBA... is symmetric and achiral.

Extensions to multidirectional lattices are possible, but are not constrained to follow some Euclidean-like structure, such as nodes in crystal lattices.

\subsection{Matrices}

According to our definition of symmetry [13], a rectangular matrix $\mathbf{A}$ of $m$ lines and $p$ columns is viewed as a function of a bicomponent index $x$ such that the first component of $x$ takes integer values in $\{1, \ldots, m\}$ and the second component of $x$ takes integer values in $\{1, \ldots, p\}$. The $m p$ values returned by the function are the $m p$ elements of the matrix, and are not necessarily numbers: they may be of any type. The distance between two bicomponent indices $x_{1}$ and $x_{2}$ is issued from the ordinary Euclidean norm, but applies here to two ordered pair of numbers: $\delta\left(x_{1}, x_{2}\right)=\left\|x_{2}-x_{1}\right\|$. So, the group $F$ contains the following isometries: $\left(\mathbf{I}_{m}, \mathbf{I}_{p}\right),\left(\mathbf{I}_{m}, \mathbf{J}_{p}\right),\left(\mathbf{J}_{m}, \mathbf{I}_{p}\right)$, and $\left(\mathbf{J}_{m}, \mathbf{J}_{p}\right)$, where $\mathbf{I}_{m}$ and $\mathbf{I}_{p}$ are the identity permutations matrices of respective sizes $m$ and $p$, and $\mathbf{J}_{m}$ and $\mathbf{J}_{p}$ are permuting respectively the lines $i$ and $m+1-i$ for $i=1, \ldots, m$ and the columns $j$ and $p+1-j$ for $j=1, \ldots, p$. In matricial form, the images of $\mathbf{A}$ through these isometries are respectively $\mathbf{I}_{m} \mathbf{A} \mathbf{I}_{p}^{\prime}, \mathbf{I}_{m} \mathbf{A} \mathbf{J}_{p}^{\prime}, \mathbf{J}_{m} \mathbf{A} \mathbf{I}_{p}^{\prime}$, and $\mathbf{J}_{m} \mathbf{A} \mathbf{J}_{p}^{\prime}$. So $F^{+}=\left\{\left(\mathbf{I}_{m}, \mathbf{I}_{p}\right)\right\}$, and $F^{-}=\left\{\left(\mathbf{I}_{m}, \mathbf{J}_{p}\right),\left(\mathbf{J}_{m}, \mathbf{I}_{p}\right),\left(\mathbf{J}_{m}, \mathbf{J}_{p}\right)\right\}$. The three elements of $F^{-}$are mirrors, any of them being the product of the two other ones. In the Euclidean case, the product of two mirrors would not be a mirror. In this particular case, $F$ is a commutative group.

When $p=m, \mathbf{A}$ is a square matrix, and $F^{-}$contains three more mirrors. One corresponds to the usual matrix transposition (i.e. lines and columns are exchanged), and the three other ones correspond to the respective products of the transposition by $\left(\mathbf{I}_{m}, \mathbf{J}_{m}\right),\left(\mathbf{J}_{m}, \mathbf{I}_{m}\right)$, and $\left(\mathbf{J}_{m}, \mathbf{J}_{m}\right)$. Again, all products in $F$ are commutative: $F$ is a commutative group. The product of the transposition by $\left(\mathbf{J}_{m}, \mathbf{J}_{m}\right)$ is the transposition through the antidiagonal of $\mathbf{A}$. A matrix called symmetric in the usual sense, i.e. identical to its transpose, is thus achiral. A matrix usually said to be "non-symmetric" may be in fact either chiral or achiral (and thus symmetric), even if it is rectangular, depending of its structure.

It is pointed out that a matrix should not be confused with a rectangle or a square in the Euclidean plane, despite that we conventionnally draw matrices rectangularly: e.g., the mirror $\left(\mathbf{J}_{m}, \mathbf{J}_{p}\right)$ should not be confused with the center of symmetry of a rectangle in the Euclidean plane, which is associated to a rotation of angle $\pi$, this latter being not a mirror. In fact, matrices and tensors are not geometrical figures, and when existing, their symmetry properties should not be confused with those of their graphical representations.

\subsection{Graphs}

Graphs are nodes and edges structures of major importance in many areas, including mathematics, chemistry, econometrics, etc. According to [13], the isometries asso- 
ciated to a graph of $m$ nodes are the $m$ ! possible renumbering of the nodes, each renumbering being represented by a permutation of size $m$. The group $F$ is the group of these $m$ ! permutations. The identity permutation is noted $I$. Each permutation $P$ can be decomposed into $k$ independent cycles (i.e. disjoint cycles) of lengths $m_{1}, \ldots, m_{k}$, with $m_{1}+\cdots+m_{k}=m$. To each cycle is associated a circular permutation $C_{i}$ of length $m_{i}, i=1, \ldots, k$. Then, $P=C_{1} C_{2} \ldots C_{k}$, and all elements of this latter product commute, although the product of permutations does not commute, in general. The smallest integer $K$ such that $P^{K}=I$ is the least common multiple of $m_{1}, \ldots, m_{k}$. A permutation containing exactly one cycle of length 2 is usually called a transposition, and is a mirror. Except $I$, all permutations $P$ composed of cycles of length 1 or 2 are mirrors. More generally, we have Theorem 3:

\section{Theorem $3 P \in F^{+}$if and only if all cycles of $P$ have an odd length.}

Proof If $P$ has at least one cycle $C_{i}$ of even length $m_{i}$, it can be written $P=\Pi C_{i} \Pi C_{j}$, where $\Pi C_{j}$ is the product of odd length cycles (contains at least $I$ ). Assuming $P$ in $F^{+}$would mean that $\Pi C_{i}$ would be in $F^{+}$because each cycle of odd length $m_{j}$ is a square, i.e. $C_{j}=C j^{\left(m_{j}+1\right) / 2} C j^{\left(m_{j}+1\right) / 2}$, and so the product $\Pi C_{i}=P\left(\Pi C_{j}\right)^{-1}$ would fall in $F^{+}$. But the determinant of each $C_{i}$ is -1 , so that none of the $C_{i}$ is in $F^{+}$. If there is only one even length cycle $C_{i}$ in $P$, then $C_{i} \in F^{-}$, and thus $P \in F^{-}$. If there are at least two even length cycles in the expression of $P$, each of these even length cycles $C_{i}$ can be written as product of other permutations, but since the $C_{i}$ are independent, none of the permutations involved in the expression of one of the even length cycles can appear in the expression of an other one of the even length cycles, except the identity. So the product of the $C_{i}$ cannot exhibit any square apart $I$, and thus cannot be in $F^{+}$unless $P=I$, in which case $P$ has only cycles of odd lengths 1 .

In order to exemplify symmetry in graphs, we consider the graphs associated to some molecular structural formulas. E.g. the molecular graph of the water $\mathrm{H}-\mathrm{O}-\mathrm{H}$ contains three nodes and two edges. There are two automorphisms: one is associated to the identity permutation of order 3 , and the other one is associated to a permutation exchanging the two hydrogens and leaving invariant the oxygen. This latter permutation is a mirror, and so the graph of the water is achiral. It is emphazised here that this achirality should not be confused with the geometrical achirality of the water molecule. The graph of the hydrochloric acid $\mathrm{H}-\mathrm{Cl}$ is chiral because it has only one automorphism (the identity), although it is geometrically achiral. The graph of the methanol $\mathrm{CH}_{3}-\mathrm{O}-\mathrm{H}$ has 6 automorphisms, which constitute the subgroup isomorphic to all permutations of 3 elements (the hydrogens of the methyl group): this graph offers both direct and indirect symmetry (and so it is achiral). The hydrogen suppressed molecular graph of any linear alcane containing $n$ carbons $(n \geq 2), \mathrm{C}-\mathrm{C}-\cdots-\mathrm{C}-\mathrm{C}$, has two automorphisms and is achiral.

Outside the field of chemistry, a graph containing $m p$ nodes $(m \geq 2, p>2)$ constituting a single ring of size $m p$ containing cyclically $m$ repetitions of the same ordered sequence of $p$ different nodes (e.g. $p$ different letters), has a symmetry group of $m$ automorphisms which is isomorphic to the subgroup of all circular permutations of $m$ elements and to $(\mathbb{Z} / m \mathbb{Z},+)$. When $m$ is even, the graph has both direct and indirect symmetry. When $m$ is odd, the graph has direct symmetry and is chiral. 


\section{Discussion and conclusion}

The main goal of this paper was to show that the concept of chirality/achirality is not a prerogative of Euclidean spaces. We have demonstrated that the existence of chirality or achirality is induced by the group structure of the isometries. This group of isometries is assumed to operate on a metric space rather than only on Euclidean spaces. This latter is a metric space, too, but all other requirements needed to build a Euclidean space are relaxed: we work under weaker assumptions than in the Euclidean case, and thus it is a major progress. Furthermore, we have proved that our definition of chirality/achirality is indeed equivalent to the usual one in the Euclidean case. However, the usual sense of chirality involving orientation of space looses its original meaning: this is fortunate, because space orientation is undefined when the space is not Euclidean.

A practical consequence of our definition of chirality is that we can classify the symmetry operators of an object into two classes: the direct symmetry operators, and the indirect symmetry operators, which induce achirality. So, depending of its symmetry operators, a symmetric object can be either direct-symmetric and not indirect-symmetric (i.e. chiral), or indirect-symmetric (i.e. achiral) and not directsymmetric, or both direct and indirect symmetric. This classification works in the non-Euclidean case, but in this latter situation, we must keep in mind that we are dealing with objects in a space which is not Euclidean. It means that when we superimpose this model of symmetry to a Euclidean model, each model of symmetry induces its own properties to the object. E.g., a graph for which the nodes receive cartesian coordinates has the symmetries due to the graph automorphisms, and has the Euclidean symmetries due to its geometrical representation. The symmetry groups of these two kinds of symmetries are not isomorphic, in general. If we would consider together these both kinds of symmetries, we would have to metrize the cartesian product of the Euclidean space by the metric space underlying the graph structure. A similar situation occurs in chemistry, where both the geometry of the molecule and the graph associated to the structural formula must be considered together. E.g. the bromo-chloro-fluoromethane $\mathrm{Br}-\mathrm{CHF}-\mathrm{Cl}$, assuming that the carbon is at the center of a regular tetrahedron with each of the four other atoms lying at its vertices, is achiral in the Euclidean space, has a chiral graph (only one automorphism), and the whole molecule is chiral if we consider both the graph and the spatial geometry. Other examples of symmetry in product spaces have been presented [10].

An other consequence of our definition of chirality is based on the partition of the group $F$ of isometries into two subsets, i.e. the direct subgroup $F^{+}$and its complement $F^{-}=F \backslash F^{+}$. The neutral element is always in $F^{+}$, and this fact enlights why the problems of building direct symmetry measures and building chirality measures may require different solutions (see section 6 in [11]). The full discussion of symmetry measures being outside the scope of the present work, the reader is referred to the cited paper.

Several situations of interest have not been considered, such as hyperbolic spaces and Thurston geometries $[7-9,16,18]$. These geometries will be considered in a subsequent paper. 
Acknowledgements Many thanks to Antonio Mucherino and Carlile Lavor for their kind invitation to consider this paper in the special issue dedicated to the memory of Michel Deza. I am highly grateful to György Darvas, CEO of Symmetrion, for permission of reproduction of the content of this paper. I thank very much the anonymous reviewers for their kind help to improve the content of the paper.

Open Access This article is distributed under the terms of the Creative Commons Attribution 4.0 International License (http://creativecommons.org/licenses/by/4.0/), which permits unrestricted use, distribution, and reproduction in any medium, provided you give appropriate credit to the original author(s) and the source, provide a link to the Creative Commons license, and indicate if changes were made.

\section{Appendix: Factorisation of rotations}

Theorem 4 Any d-dimensional rotation can be written as a product of $d(d-1) / 2$ Givens rotations.

Proof Let $\mathbf{R}$ be a $d$-dimensional rotation matrix, $d>1$. A Givens rotation is represented by a matrix of the form:

$$
\mathbf{G}(j, k, \theta)=\left(\begin{array}{lllll}
I & 0 & 0 & 0 & 0 \\
0 & c & 0 & -s & 0 \\
0 & 0 & I & 0 & 0 \\
0 & s & 0 & c & 0 \\
0 & 0 & 0 & 0 & I
\end{array}\right)
$$

where $c=\cos \theta$ and $s=\sin \theta$ appear at the intersections jth and kth rows and columns, $1 \leq j<k \leq d$, and the square blocks $\mathbf{I}$ are identity matrices of respective sizes $j-1$, $k-1-j$, and $d-k$. Since the multiplication of any matrix of $d$ lines by $\mathbf{G}(j, k, \theta)$ on the left affects only the lines $j$ and $k$, we restrict our attention to these lines. Given the reals $a$ and $b$, we can set $\theta$ in order to have:

$$
\left(\begin{array}{ll}
c & -s \\
s & c
\end{array}\right)\left(\begin{array}{l}
a \\
b
\end{array}\right)=\left(\begin{array}{l}
r \\
0
\end{array}\right)
$$

Practically, we set $s=-b / \sqrt{a^{2}+b^{2}}$ and $c=a / \sqrt{a^{2}+b^{2}}$, and we get $r=$ $\sqrt{a^{2}+b^{2}}$. Applying successively Givens rotations to the $d(d-1) / 2$ couples $(j, k)$, $j=1, \ldots, d-1, k=j+1, \ldots, d$, we factorize $\mathbf{R}$ as a product of $d(d-1) / 2$ Givens rotations matrices by an upper triangular matrix $\mathbf{U}$ (this is a particular case of the well-known QR decomposition). $\mathbf{U}$ is itself a rotation matrix because $\mathbf{R}$ is a rotation matrix. So, the diagonal elements of $\mathbf{U}$ can contain only +1 and -1 values, and thus $\mathbf{U}$ is diagonal because its columns are unit vectors. Since the process generates a non negative value at each intersection of the line $j$ and the column $j$ for $j=1, \ldots, d-1$, the $d-1$ first diagonal elements of $\mathbf{U}$ take the value +1 , and its last diagonal element receives the value of the determinant of $\mathbf{R}$, i.e. +1 because $\mathbf{R}$ is a rotation matrix. Thus, $\mathbf{U}=\mathbf{I}$ and $\mathbf{R}$ is factorized as the product of $d(d-1) / 2$ Givens rotations matrices.

Corollary 1 Any rotation can be expressed as a product of $d(d-1) / 2$ squares of Givens rotations.

Proof $\mathbf{G}(j, k, \theta)=\mathbf{G}(j, k, \theta / 2) \mathbf{G}(j, k, \theta / 2)$. Then apply Theorem 4. 
Remark: in the literature, the Givens rotations matrices are often defined as being the transposed of the ones above. This difference is meaningless in our context. We just notice that when $d=2$, the Givens rotation matrix of angle $\theta$ we used here corresponds to a rotation of angle $+\theta$ when operating on the left of a column vector, which is the standard way to write this kind of product. The transposed rotation matrix corresponds to a rotation of angle $-\theta$.

\section{References}

1. Coppersmith, D., Petitjean, M.: About the optimal density associated to the chiral index of a sample from a bivariate distribution. C. R. Acad. Sci. Paris Sér. I 340(8), 599-604 (2005)

2. Darvas, G.: Symmetry. Birkhäuser, Basel (2007)

3. Deza, E., Deza, M.M.: Dictionary of Distances. Elsevier, Amsterdam (2006)

4. Deza, M., Schulte, E.: Editorial on Tessellations. Symmetry Cult. Sci. 22(1-2), 5 (2011)

5. Deza, M., Petitjean, M., Markov, K. (eds.) Mathematics of Distances and Applications. Proceedings of the MDA 2012 Conference. ITHEA Publication, Sofia (2012)

6. Deza, M.M., Deza, E.: Encyclopedia of Distances, 4th edn. Springer, Berlin (2016)

7. Molnár, E.: The projective interpretation of the eight 3-dimensional homogeneous geometries. Beitr. Algebra Geom. 38(2), 261-288 (1997)

8. Molnár, E.: Combinatorial construction of tilings by barycentric simplex orbits ( $D$ symbols $)$ and their realizations in Euclidean and other homogeneous spaces. Acta Crystallogr. A61(6), 542-552 (2005)

9. Molnár, E., Szirmai, J.: On Nil crystallography. Symmetry Cult. Sci. 17(1-2), 55-74 (2006)

10. Petitjean, M.: Chiral mixtures. J. Math. Phys. 43(8), 4147-4157 (2002)

11. Petitjean, M.: Chirality and symmetry measures: a transdisciplinary review. Entropy 5(3), 271-312 (2003)

12. Petitjean, M.: From shape similarity to shape complementarity: toward a docking theory. J. Math. Chem. 35(3), 147-158 (2004)

13. Petitjean, M.: A definition of symmetry. Symmetry Cult. Sci. 18(2-3), 99-119 (2007). https://hal. archives-ouvertes.fr/hal-01552499

14. Petitjean, M.: Chirality in metric spaces. Symmetry Cult. Sci. 21(1-3), 27-36 (2010)

15. Petitjean, M.: The most chiral disphenoid. MATCH Commun. Math. Comput. Chem. 73(2), 375-384 (2015)

16. Scott, P.: The geometries of 3-manifolds. Bull. Lond. Math. Soc. 15, 401-487 (1983)

17. Thomson, W.: Baltimore Lectures on Molecular Dynamics and the Wave Theory of Light, Appendix H., Sect. 22, Footnote p. 619. Cambridge University Press Warehouse, London (1904)

18. Thurston, W.P.: Three-Dimensional Geometry and Topology, vol. 1. Princeton University Press, Princeton (1997)

19. Weyl, H.: Symmetry. Princeton University Press, Princeton (1952) 